\title{
Long-term risk of type 2 diabetes mellitus in relation to BMI and weight change among women with a history of gestational diabetes mellitus: a prospective cohort study
}

\author{
Wei Bao • Edwina Yeung • Deirdre K. Tobias • Frank B. Hu • Allan A. Vaag • \\ Jorge E. Chavarro • James L. Mills • Louise G. Grunnet • Katherine Bowers • \\ Sylvia H. Ley • Michele Kiely • Sjurdur F. Olsen • Cuilin Zhang
}

Received: 27 October 2014 / Accepted: 5 February 2015 / Published online: 22 March 2015

(C) Springer Verlag (outside the USA) 2015

\begin{abstract}
Aims/hypothesis Women with a history of gestational diabetes mellitus (GDM) are advised to control their weight after pregnancy. We aimed to examine how adiposity and weight change influence the long-term risk of developing type 2 diabetes after GDM.

Methods We included 1,695 women who had incident GDM between 1991 and 2001, as part of the Diabetes \& Women's Health study, and followed them until the return of the 2009 questionnaire. Body weight and incident type 2 diabetic cases were reported biennially. We defined baseline as the questionnaire period when women reported an incident GDM pregnancy. We estimated HRs and 95\% CIs using Cox proportional hazards models.
\end{abstract}

Electronic supplementary material The online version of this article (doi:10.1007/s00125-015-3537-4) contains peer-reviewed but unedited supplementary material, which is available to authorised users.

W. Bao $\cdot$ E. Yeung $\cdot$ J. L. Mills $\cdot$ M. Kiely $\cdot$ C. Zhang $(\bowtie)$

Epidemiology Branch, Division of Intramural Population Health Research, Eunice Kennedy Shriver National Institute of Child Health and Human Development, National Institutes of Health, 6100

Executive Boulevard, Rockville, MD 20852, USA

e-mail: zhangcu@mail.nih.gov

D. K. Tobias · F. B. Hu • J. E. Chavarro • S. H. Ley

Department of Nutrition, Harvard T.H. Chan School of Public

Health, Boston, MA, USA

D. K. Tobias

Division of Preventive Medicine, Brigham and Women's Hospital and Harvard Medical School, Boston, MA, USA

F. B. Hu • J. E. Chavarro

Department of Epidemiology, Harvard T.H. Chan School of Public

Health, Boston, MA, USA
Results We documented 259 incident cases of type 2 diabetes during up to 18 years of follow-up. The adjusted HRs of type 2 diabetes associated with each $1 \mathrm{~kg} / \mathrm{m}^{2}$ increase in BMI were $1.16(95 \%$ CI $1.12,1.19)$ for baseline BMI and $1.16(95 \% \mathrm{CI}$ $1.13,1.20)$ for most recent BMI. Moreover, each $5 \mathrm{~kg}$ increment of weight gain after GDM development was associated with a $27 \%$ higher risk of type 2 diabetes (adjusted HR 1.27; $95 \%$ CI 1.04, 1.54). Jointly, women who had a BMI $\geq 30.0 \mathrm{~kg} /$ $\mathrm{m}^{2}$ at baseline and gained $\geq 5 \mathrm{~kg}$ after GDM had an adjusted HR of 43.19 (95\% CI 13.60, 137.11), compared with women who had a BMI $<25.0 \mathrm{~kg} / \mathrm{m}^{2}$ at baseline and gained $<5 \mathrm{~kg}$ after GDM.

Conclusions/interpretation Baseline BMI, most recent BMI and weight gain after GDM were significantly and positively

F. B. Hu • J. E. Chavarro

Channing Division of Network Medicine, Department of Medicine, Brigham and Women's Hospital and Harvard Medical School, Boston, MA, USA

A. A. Vaag • L. G. Grunnet

Department of Endocrinology, Rigshospitalet,

Copenhagen, Denmark

K. Bowers

Division of Biostatistics and Epidemiology, Department of

Pediatrics, Cincinnati Children's Hospital Medical Center,

Cincinnati, OH, USA

S. F. Olsen

Centre for Fetal Programming, Department of Epidemiology

Research, Statens Serum Institut, Copenhagen, Denmark 
associated with risk of progression from GDM to type 2 diabetes.

Keywords BMI - Gestational diabetes mellitus - Type 2 diabetes mellitus $\cdot$ Weight change

$\begin{array}{ll}\text { Abbreviations } \\ \text { ADA } & \text { American Diabetes Association } \\ \text { AHEI } & \text { Alternate Healthy Eating Index } 2010 \\ \text { DPP } & \text { Diabetes Prevention Program } \\ \text { FFQ } & \text { Food frequency questionnaire } \\ \text { GDM } & \text { Gestational diabetes mellitus } \\ \text { NDDG } & \text { National Diabetes Data Group } \\ \text { NDEP } & \text { National Diabetes Education Program } \\ \text { NHS } & \text { Nurses' Health Study }\end{array}$

\section{Introduction}

Gestational diabetes mellitus (GDM) is a common pregnancy complication defined as glucose intolerance with onset or first recognition during pregnancy [1]. Women who develop GDM during pregnancy probably have reduced insulin secretion and/or chronic insulin resistance before pregnancy [2, 3]. As a result, they are at substantially increased risk of developing type 2 diabetes later in life [4]. One-third of women with type 2 diabetes were found to have a history of GDM [5]. The increasing prevalence of GDM [6-8] parallels the worldwide epidemic of type 2 diabetes [9]. Women with a history of GDM represent a 'revealed' high-risk group for type 2 diabetes [10], because GDM cases are routinely identified through blood glucose testing between 24 and 28 weeks of gestation [1]. It remains to be elucidated whether the natural course of the progression from GDM to overt type 2 diabetes is significantly altered by lifestyle factors.

Excess adiposity and weight gain are well-documented risk factors of type 2 diabetes in the general population [11-15]. Compared with the general population, women who develop GDM are more likely to be overweight or obese at the time of GDM diagnosis and are more likely to develop incident overweight or obesity in later life [16]. As a result, women with a history of GDM are usually advised to control their weight after delivery $[1,16]$. The National Diabetes Education Program (NDEP) recently called for women with a history of GDM to be referred to a registered dietitian or community programme for weight management [17]. A similar recommendation was also given by the American Diabetes Association (ADA) [1]. Despite these recommendations, weight gain is common among women with a history of GDM after the index pregnancy and throughout their lifespan $[16,18]$. The long-term risk of type 2 diabetes in association with adiposity and weight change among women with a history of GDM has not been clearly characterised [19].

In this study, we used data from the Nurses' Health Study (NHS) II cohort to examine the associations of BMI at baseline and follow-up, as well as weight change after GDM, with long-term risk of type 2 diabetes among women with a history of GDM.

\section{Methods}

Study population The study population was composed of women with a history of GDM in NHS II, as part of the ongoing Diabetes \& Women's Health study [20], which aims to identify determinants of the progression from GDM to type 2 diabetes. NHS II, established in 1989, is an ongoing prospective cohort study of 116,671 female nurses aged 25 44 years at study initiation [21]. Participants receive a biennial questionnaire to update information on health-related behaviours and disease outcomes. The follow-up rate for each questionnaire cycle was greater than $90 \%$ up to 2009 . This study was approved by the Partners Human Research Committee (Boston, MA, USA), with participants' consent implied by the return of the questionnaires.

Women were eligible for inclusion if they reported incident GDM from 1991 to 2001. They were followed up until the return of the 2009 questionnaire. The 2001 questionnaire was the last time questions regarding GDM were included, as the majority of NHS II participants had passed reproductive age by then. In a prior validation study, $94 \%$ of self-reported GDM cases were confirmed by medical records [21]. In a random sample of parous women without GDM, 83\% reported a glucose screening test during pregnancy and $100 \%$ reported frequent prenatal urine screening, suggesting a high level of GDM surveillance in this cohort [21]. We excluded women from the analytical population if they had: (1) prevalent GDM in 1989; (2) type 2 diabetes, cardiovascular disease or cancer prior to their GDM pregnancy or before the return of their first post-GDM questionnaire; or (3) a multiple gestation (twins or triplets). Electronic supplementary material (ESM) Fig. 1 briefly outlines the data collection and follow-up of the study population.

Assessment of exposures The NHS II participants reported their weight and height in the 1989 questionnaire and they further updated their current weight on each biennial questionnaire. Only $2-4 \%$ of the study participants who responded to the questionnaire had missing data on body weight across the follow-up years. Self-reported weight was highly correlated with technician-measured weight $(r=0.97)$ among a subset of NHS cohort participants [22]. BMI was computed as weight in kilograms divided by the square of height in metres $\left(\mathrm{kg} / \mathrm{m}^{2}\right)$. We classified the participants' BMI into the 
categories $<18.5,18.5-24.9,25.0-29.9,30.0-34.9,35.0-39.9$ and $\geq 40.0 \mathrm{~kg} / \mathrm{m}^{2}$, corresponding to definitions of underweight, normal weight, overweight, class I obesity, class II obesity and class III obesity, respectively, according to National Institutes of Health guidelines [23]. Since only $1 \%$ of the participants had a BMI $<18.5 \mathrm{~kg} / \mathrm{m}^{2}$, we merged this category and $18.5-24.9 \mathrm{~kg} / \mathrm{m}^{2}$ into BMI $<25.0 \mathrm{~kg} / \mathrm{m}^{2}$ to ensure statistical power in the present analysis.

Information on age, smoking status, age at first birth, reproductive history, oral contraceptive use and menopausal status was biennially updated from 1989. Parity was defined as the number of pregnancies lasting greater than 6 months. Race/ethnicity data were collected in 1989, and family history of diabetes was collected in 1989 and updated in 2005. Diet information was updated every 4 years since 1991 using a semi-quantitative food frequency questionnaire (FFQ). The FFQ is designed to measure average dietary intake over the past year and has been extensively validated [24-26]. To characterise overall diet quality, we created a summary diet score in terms of the Alternate Healthy Eating Index 2010 (AHEI), as previously described [27]. Total physical activity was ascertained in 1991, 1997 and every 4 years thereafter by frequency of engaging in common recreational activities, from which metabolic equivalent task-hours per week were derived. The questionnaire-based estimates correlated well with detailed activity diaries in a prior validation study $(r=0.56)$ [28].

Ascertainment of outcome Participants reporting physiciandiagnosed type 2 diabetes on each biennial questionnaire were mailed a supplemental questionnaire regarding symptoms, diagnostic tests and hypoglycaemic therapy to confirm selfreported diagnoses. Confirmed diabetes required at least one of the following reported on the supplementary questionnaire according to ADA criteria [29]: (1) one or more classic symptoms (excessive thirst, polyuria, weight loss, hunger, pruritus or coma) plus elevated glucose levels (fasting plasma glucose concentration $\geq 7.0 \mathrm{mmol} / 1$ or random plasma glucose $\geq 11.1 \mathrm{mmol} / \mathrm{l}$ ); or (2) no symptoms reported but two or more elevated plasma glucose concentrations on more than one occasion (fasting $\geq 7.0 \mathrm{mmol} / \mathrm{l}$, random $\geq 11.1 \mathrm{mmol} / \mathrm{l}, 2 \mathrm{~h}$ OGTT $\geq 11.1 \mathrm{mmol} / \mathrm{l}$ ); or (3) treatment with insulin or an oral hypoglycaemic agent. Before 1998, fasting plasma glucose $\geq 7.8 \mathrm{mmol} / 1$ was used instead of $\geq 7.0 \mathrm{mmol} / 1$ for the diagnosis of diabetes according to the criteria of the National Diabetes Data Group (NDDG) [30]. In a previous validation study [31], high accuracy (98\%) was observed comparing our classification with medical records according to NDDG criteria. In a second validation study conducted in 1999, the diagnosis of type 2 diabetes was confirmed by medical record reviews according to ADA criteria, with high validity (99\%).

Statistical analysis In this analysis, baseline was defined as the questionnaire period when women reported an incident
GDM pregnancy during 1991-2001. Therefore, baseline weight was within 2 years after GDM diagnosis. Weight change since baseline was defined as the most recent body weight at follow-up minus baseline body weight. We computed follow-up time from the date of diagnosis of GDM to the date of diagnosis of type 2 diabetes, death, last biennial questionnaire response or return of the 2009 questionnaire, whichever came first. Updating of exposure status ceased if a participant reported a diagnosis of chronic disease (e.g. cardiovascular disease, cancer), because such diagnoses may lead to unintentional or intentional changes in body weight. If exposure data were missing in one questionnaire cycle, the values were carried forward from the previous questionnaire for which the data were captured. However, missing exposure data were not carried forward and were therefore excluded in the analysis of weight change.

We estimated HRs and 95\% CIs using Cox proportional hazards models stratified by time since GDM diagnosis. We conducted tests of linear trend across categories of BMI or weight change by assigning the median value for each category and fitting this continuous variable in the models. In the multivariable analysis estimating the effect of updated BMI (i.e. most recent BMI within 2 years before diagnosis of type 2 diabetes), we adjusted for various potential confounding factors, including age, parity, age at first birth, race/ethnicity, family history of diabetes, oral contraceptive use, menopausal status, cigarette smoking, breastfeeding duration, total energy intake, diet quality (i.e. AHEI score) and physical activity. All these covariates except race/ethnicity were updated over time. For the analysis assessing baseline BMI in relation to risk of type 2 diabetes, we adjusted for the baseline measures of the above variables. To evaluate weight change after GDM in association with risk of type 2 diabetes, we adjusted for baseline BMI, the aforementioned potential confounding factors, and simultaneous changes in lifestyle factors, including smoking status (never to never, never to current, past to past, past to current, current to past, current to current), as well as baseline and changes in breastfeeding duration, parity, total energy intake, diet quality (i.e. AHEI score) and physical activity. Categorical covariates included an indicator for missing data, if necessary.

We evaluated potential effect modification by stratified analyses according to age ( $<40$ years or $\geq 40$ years), family history of diabetes (yes/no), diet quality (AHEI score, above or below the median), physical activity (above or below the median), breastfeeding duration ( $<6$ months or $\geq 6$ months) and time since GDM pregnancy ( $<10$ or $\geq 10$ years). To address the potential bias by medical surveillance for type 2 diabetes, we conducted a sensitivity analysis restricting the definition of type 2 diabetic cases to participants reporting at least one diabetic symptom at the time of diagnosis. To minimise potential bias from subclinical type 2 diabetes leading to unintentional weight loss, we conducted additional analyses in 
which we excluded women who reported type 2 diabetes in the subsequent questionnaire after reporting GDM: for example, when a woman reported GDM in 1991 and type 2 diabetes in 1993. All statistical analyses were performed using SAS software (version 9.3; SAS Institute, Cary, NC, USA). A $p$ value $<0.05$ was considered statistically significant.

\section{Results}

During up to 18 years of follow-up (18,596 person-years), we documented 259 incident cases of type 2 diabetes among 1,695 women with a history of GDM. The mean follow-up duration was 13.1 years (median 14.0 years). At baseline, women with higher BMI were older, less physically active and more likely to have a family history of diabetes. They had lower diet quality and shorter breastfeeding duration (Table 1). During follow-up, we observed an average weight gain of $0.47 \mathrm{~kg}$ per year after the index GDM pregnancy.

Both baseline and updated BMI were strongly and positively associated with future risk of type 2 diabetes. Figure 1 depicts the unadjusted cumulative incidence of type 2 diabetes according to baseline BMI. After adjustment for age, parity and other major diabetic risk factors, women who had a baseline BMI 25.0-29.9, 30.0-34.9, 35.0-39.9 and $\geq 40.0 \mathrm{~kg} / \mathrm{m}^{2}$, compared with women having a baseline BMI $<25.0 \mathrm{~kg} / \mathrm{m}^{2}$, had adjusted HRs of 3.62 (95\% CI 2.22, 5.91), $6.72(95 \%$ CI $3.97,11.37), 15.28$ (95\% CI 8.52, 27.41) and 17.28 (95\% CI $8.63,34.60$ ), respectively ( $p$ for linear trend $<0.001$ ). When baseline BMI was modelled as a continuous variable, each $1 \mathrm{~kg} / \mathrm{m}^{2}$ increase in baseline BMI was associated with a $16 \%$ higher risk of type 2 diabetes (HR 1.16; 95\% CI 1.12, 1.19) (Table 2; ESM Fig. 2). A similar association of type 2

Table 1 Baseline characteristics of the study population, according to $\mathrm{BMI}^{\mathrm{a}, \mathrm{b}}$

\begin{tabular}{|c|c|c|c|c|c|}
\hline \multirow[t]{2}{*}{ Characteristic } & \multicolumn{5}{|c|}{ Baseline BMI $\left(\mathrm{kg} / \mathrm{m}^{2}\right)$} \\
\hline & $<25.0$ & $25.0-29.9$ & $30.0-34.9$ & $35.0-39.9$ & $\geq 40.0$ \\
\hline Number of participants & 717 & 473 & 280 & 149 & 76 \\
\hline Age, years & $39.5(5.0)$ & $39.5(5.2)$ & $40.3(5.1)$ & $40.9(4.9)$ & $42.6(4.8)$ \\
\hline Family history of diabetes, $\%$ & 18.0 & 20.7 & 24.0 & 28.7 & 32.1 \\
\hline White race, $\%$ & 90.0 & 87.9 & 92.7 & 93.2 & 97.1 \\
\hline Current use of oral contraceptives, $\%$ & 11.9 & 9.8 & 7.1 & 8.7 & 4.7 \\
\hline Current smoking, $\%$ & 6.8 & 11.2 & 7.2 & 8.9 & 10.2 \\
\hline Alcohol, g/day & $3.1(5.5)$ & $2.3(5.2)$ & $1.9(3.6)$ & $0.8(1.9)$ & $1.1(3.0)$ \\
\hline Physical activity, MET h/week & $18.4(30.3)$ & $14.8(18.4)$ & $12.1(14.3)$ & $10.0(13.4)$ & $8.1(11.9)$ \\
\hline Total energy, kJ/day ${ }^{\mathrm{a}}$ & $8,100(2,343)$ & $8,314(2,410)$ & $8,284(2,364)$ & $8,142(2,644)$ & $8,849(2,607)$ \\
\hline Carbohydrates, $\% \mathrm{E}$ & $50.8(7.6)$ & $49.7(8.2)$ & $49.2(7.6)$ & $49.1(7.3)$ & $47.3(8.2)$ \\
\hline Total protein, $\% \mathrm{E}$ & $18.8(3.3)$ & $19.3(3.5)$ & $19.3(3.4)$ & $19.6(3.6)$ & $19.3(3.8)$ \\
\hline Total fat, $\% \mathrm{E}$ & $31.1(6.1)$ & $31.9(6.4)$ & $32.4(5.9)$ & $32.5(6.1)$ & $34.2(6.5)$ \\
\hline SFA, $\% \mathrm{E}$ & $10.8(2.6)$ & $11.1(2.7)$ & $11.4(2.5)$ & $11.4(2.4)$ & $12.3(2.9)$ \\
\hline MUFA, \%E & $12.1(2.8)$ & $12.4(3.0)$ & $12.6(2.6)$ & $12.5(2.7)$ & $13.1(2.8)$ \\
\hline PUFA, \%E & $5.4(1.5)$ & $5.5(1.4)$ & $5.5(1.4)$ & $5.6(1.4)$ & $5.6(2.0)$ \\
\hline trans-Fat, $\% \mathrm{E}$ & $1.5(0.6)$ & $1.6(0.6)$ & $1.8(0.6)$ & $1.8(0.6)$ & $1.9(0.7)$ \\
\hline Cereal fibre, $\mathrm{g} /$ day $^{\mathrm{b}}$ & $6.0(2.8)$ & $5.8(2.4)$ & $5.7(2.7)$ & $5.7(2.3)$ & $5.3(2.2)$ \\
\hline Glycaemic index ${ }^{\mathrm{b}}$ & $53.4(3.6)$ & $53.4(3.6)$ & $53.3(3.5)$ & $54.1(3.6)$ & $53.9(3.2)$ \\
\hline Glycaemic load $^{\mathrm{b}}$ & $122.0(22.1)$ & $119.5(23.2)$ & $117.9(21.7)$ & $119.6(20.8)$ & $114.8(23.4)$ \\
\hline Breastfeeding, months & $6.3(7.0)$ & $5.4(5.7)$ & $4.8(5.8)$ & $3.8(5.3)$ & $3.4(4.8)$ \\
\hline AHEI score & $50.1(11.8)$ & $48.1(10.8)$ & $46.6(10.6)$ & $45.0(10.0)$ & $42.2(10.2)$ \\
\hline
\end{tabular}

Values are means (SD) unless otherwise specified

Baseline was defined as the questionnaire cycle when incident GDM was first reported. The following comparisons across categories of baseline BMI were significant: age, family history of diabetes, current use of oral contraceptives, alcohol intake, physical activity, intakes of total fat, SFA, MUFA, PUFA, trans-fat and cereal fibre, glycaemic load, breastfeeding and AHEI score

${ }^{a}$ Using the conversion factor $1 \mathrm{kcal}=4.184 \mathrm{~kJ}$, total energy intakes across categories of baseline BMI were 1,936 (560), 1,987 (576), 1,980 (565), 1,946 (632) and 2,115 (623) kcal/day

${ }^{\mathrm{b}}$ Values are energy-adjusted

$\% \mathrm{E}, \%$ of energy; MET, metabolic equivalent; MUFA, monounsaturated fatty acids; PUFA, polyunsaturated fatty acids; SFA, saturated fatty acids 


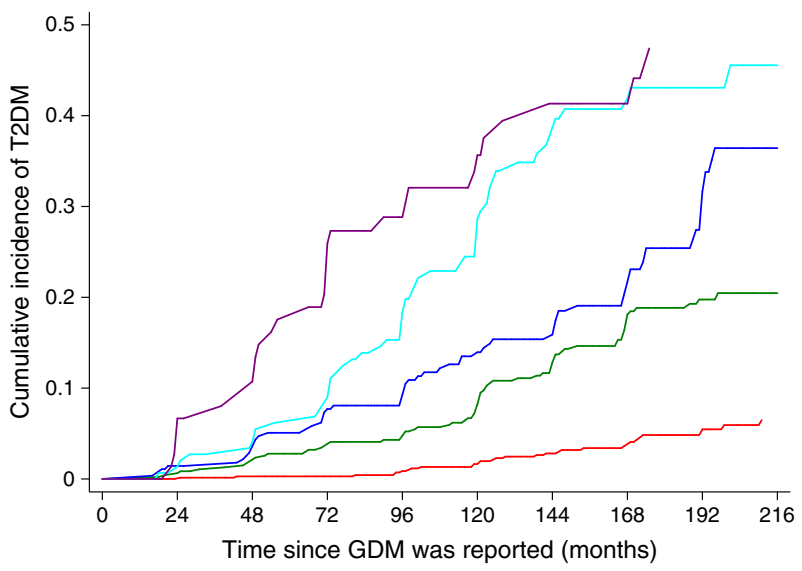

Fig. 1 Unadjusted cumulative incidence of type 2 diabetes, according to baseline BMI, among women with a history of GDM. Red line, BMI $<25.0 \mathrm{~kg} / \mathrm{m}^{2}$; green line, BMI 25.0 $29.9 \mathrm{~kg} / \mathrm{m}^{2}$; blue line, BMI $30.0-34.9 \mathrm{~kg} / \mathrm{m}^{2}$; cyan line, BMI $35.0-39.9 \mathrm{~kg} / \mathrm{m}^{2}$; purple line, BMI $\geq 40.0 \mathrm{~kg} / \mathrm{m}^{2}$

diabetic risk was observed for updated BMI. Women with an updated BMI of 25.0-29.9, 30.0-34.9, 35.0-39.9 and $\geq 40.0 \mathrm{~kg} / \mathrm{m}^{2}$, compared with women with an updated BMI of $<25.0 \mathrm{~kg} / \mathrm{m}^{2}$, had adjusted HRs of $3.82(95 \%$ CI 2.08 , 7.03), 9.69 (95\% CI 5.17, 18.16), 14.68 (95\% CI 7.47, 28.88 ) and 25.97 (95\% CI 12.62, 53.44), respectively. The adjusted HR of type 2 diabetes associated with each increment of $5 \mathrm{~kg} / \mathrm{m}^{2}$ in updated BMI was 1.16 (95\% CI 1.13, 1.20).

Weight gain after GDM was significantly associated with risk of type 2 diabetes (Table 3). After adjustment for baseline
BMI and other major diabetic risk factors, the adjusted HRs of type 2 diabetes associated with weight gain of 2.6-4.9, 5.0 9.9 and $\geq 10.0 \mathrm{~kg}$ were 1.17 (95\% CI $0.53,2.56), 1.48(95 \% \mathrm{CI}$ $0.72,3.03)$ and $1.99(95 \%$ CI $0.94,4.25)$, respectively ( $p$ for linear trend $=0.02$ ), compared with maintaining a stable body weight $( \pm 2.5 \mathrm{~kg})$ since baseline. An increment of $5 \mathrm{~kg}$ weight gain after GDM development was associated with a $27 \%$ higher risk of type 2 diabetes (HR 1.27; 95\% CI 1.04, 1.54). In the analysis of joint effect of baseline BMI and subsequent weight gain on risk of type 2 diabetes, women who had a BMI $\geq 30.0 \mathrm{~kg} / \mathrm{m}^{2}$ at baseline and gained $\geq 5 \mathrm{~kg}$ after GDM had an adjusted HR of 43.19 (95\% CI 13.60, 137.11), compared with women who had a BMI $<25.0 \mathrm{~kg} / \mathrm{m}^{2}$ at baseline and gained $<5 \mathrm{~kg}$ after GDM (Fig. 2).

The positive associations of BMI and weight change with risk of type 2 diabetes persisted across different categories of age, family history of diabetes, diet quality, physical activity, breastfeeding duration and time since GDM pregnancy. Moreover, the observed associations were robust in multiple sensitivity analyses. First, to minimise potential bias from subclinical type 2 diabetes before GDM diagnosis, we conducted a sensitivity analysis by excluding women who reported type 2 diabetes in the next questionnaire after reporting GDM, and found that the multivariable-adjusted HRs across categories of BMI or weight change were not appreciably changed. Second, sensitivity analyses restricted to women reporting at least one symptom of diabetes at diagnosis yielded similar results to those for all cases.

Table 2 Risk of type 2 diabetes among women with a history of GDM according to BMI category

\begin{tabular}{|c|c|c|c|c|c|c|c|}
\hline \multirow[t]{2}{*}{ Variable } & \multicolumn{5}{|c|}{ BMI $\left(\mathrm{kg} / \mathrm{m}^{2}\right)$} & \multirow{2}{*}{$\begin{array}{l}\text { Per } 1 \mathrm{~kg} / \mathrm{m}^{2} \\
\text { increase }\end{array}$} & \multirow{2}{*}{$\begin{array}{l}p \text { value for } \\
\text { trend }\end{array}$} \\
\hline & $<25.0$ & $25.0-29.9$ & $30.0-34.9$ & $35.0-39.9$ & $\geq 40.0$ & & \\
\hline \multicolumn{8}{|l|}{ Baseline BMI } \\
\hline Median & 22.2 & 27.4 & 32.3 & 37.1 & 43.2 & & \\
\hline Type 2 diabetic cases (n) & 31 & 75 & 67 & 56 & 30 & & \\
\hline Person-years & 8,719 & 5,172 & 2,819 & 1,347 & 539 & & \\
\hline \multicolumn{8}{|l|}{ HR $(95 \%$ CI $)$} \\
\hline Multivariable model $1^{\mathrm{a}}$ & 1.00 & $3.64(2.25,5.90)$ & $6.88(4.09,11.59)$ & $15.82(8.85,28.27)$ & $18.73(9.53,36.83)$ & $1.16(1.13,1.19)$ & $<0.001$ \\
\hline Multivariable model $2^{\mathrm{b}}$ & 1.00 & $3.62(2.22,5.91)$ & $6.72(3.97,11.37)$ & $15.28(8.52,27.41)$ & $17.28(8.63,34.60)$ & $1.16(1.12,1.19)$ & $<0.001$ \\
\hline \multicolumn{8}{|l|}{ Updated BMI } \\
\hline Median & 22.5 & 27.4 & 32.2 & 37.1 & 42.9 & & \\
\hline Type 2 diabetic cases $(n)$ & 19 & 57 & 81 & 52 & 50 & & \\
\hline Person-years & 7,403 & 5,428 & 3,323 & 1,635 & 807 & & \\
\hline \multicolumn{8}{|l|}{ HR $(95 \%$ CI) } \\
\hline Multivariable model $1^{\mathrm{a}}$ & 1.00 & $4.00(2.18,7.32)$ & $10.06(5.41,18.72)$ & $16.36(8.42,31.80)$ & $30.18(14.95,60.93)$ & $1.17(1.14,1.20)$ & $<0.001$ \\
\hline Multivariable model $2^{\mathrm{b}}$ & 1.00 & $3.82(2.08,7.03)$ & $9.69(5.17,18.16)$ & $14.68(7.47,28.88)$ & $25.97(12.62,53.44)$ & $1.16(1.13,1.20)$ & $<0.001$ \\
\hline
\end{tabular}

${ }^{\mathrm{a}}$ Multivariable model 1: adjusted for age (months), parity (1, 2,3+), age at first birth (12-24, 25-29, $\geq 30$ years), race/ethnicity (white, African-American, Hispanic, Asian, others), family history of diabetes (yes, no), oral contraceptive use (current, former, never), menopausal status (premenopausal, postmenopausal), cigarette smoking (current, former, never), breastfeeding duration $(<1,1-5,6-11, \geq 12$ months) and total energy intake (in quartiles) ${ }^{\mathrm{b}}$ Multivariable model 2: multivariable model 1 plus additional adjustment for diet quality (i.e. AHEI, in quartiles) and physical activity (in quartiles) 
Table 3 Risk of type 2 diabetes among women with a history of GDM according to weight change since baseline

\begin{tabular}{|c|c|c|c|c|c|c|c|}
\hline & \multicolumn{5}{|c|}{ Weight change since baseline $(\mathrm{kg})$} & \multirow{2}{*}{$\begin{array}{l}\text { Per } 5 \mathrm{~kg} \\
\text { increase }\end{array}$} & \multirow{2}{*}{$\begin{array}{l}p \text { value for } \\
\text { trend }\end{array}$} \\
\hline & $<-2.5$ & -2.5 to +2.5 & +2.6 to +4.9 & +5.0 to +9.9 & $\geq+10.0$ & & \\
\hline Median & -5.9 & 0 & 4.1 & 6.8 & 14.1 & & \\
\hline Type 2 diabetic cases $(n)$ & 33 & 37 & 21 & 35 & 38 & & \\
\hline Person-years & 1,870 & 4,272 & 2,061 & 2,171 & 1,553 & & \\
\hline \multicolumn{8}{|l|}{$\mathrm{HR}(95 \% \mathrm{CI})$} \\
\hline Multivariable model $1^{\mathrm{a}}$ & $0.79(0.37,1.67)$ & 1.00 & $1.14(0.53,2.48)$ & $1.49(0.74,3.01)$ & $1.93(0.93,4.00)$ & $1.26(1.04,1.53)$ & 0.02 \\
\hline Multivariable model $2^{\mathrm{b}}$ & $0.81(0.38,1.76)$ & 1.00 & $1.17(0.53,2.56)$ & $1.48(0.72,3.03)$ & $1.99(0.94,4.25)$ & $1.27(1.04,1.54)$ & 0.02 \\
\hline
\end{tabular}

${ }^{a}$ Multivariable model 1: adjusted for age (months), age at first birth (12-24, 25-29, $\geq 30$ years), race/ethnicity (white, African-American, Hispanic, Asian, others), family history of diabetes (yes, no), oral contraceptive use (current, former, never), menopausal status (premenopausal, postmenopausal), change in cigarette smoking status (never to never, never to current, past to past, past to current, current to past, current to current), parity at baseline (1,2, $3+)$, change in parity $(0,1,2+)$, and baseline and changes in breastfeeding duration $(<1,1-5,6-11, \geq 12$ months), total energy intake (in quartiles) and baseline BMI $\left(<25.0,25.0-29.9,30.0-34.9,35.0-39.9, \geq 40.0 \mathrm{~kg} / \mathrm{m}^{2}\right)$

${ }^{\mathrm{b}}$ Multivariable model 2: multivariable model 1 plus additional adjustment for baseline and changes in diet quality (i.e. AHEI, in quartiles) and physical activity (in quartiles)

\section{Discussion}

In this prospective cohort study with up to 18 years of followup, we found that both initial BMI within 2 years after diagnosis of GDM and the most recent BMI before diagnosis of type 2 diabetes were positively and strongly associated with a greater risk of type 2 diabetes among women with a history of

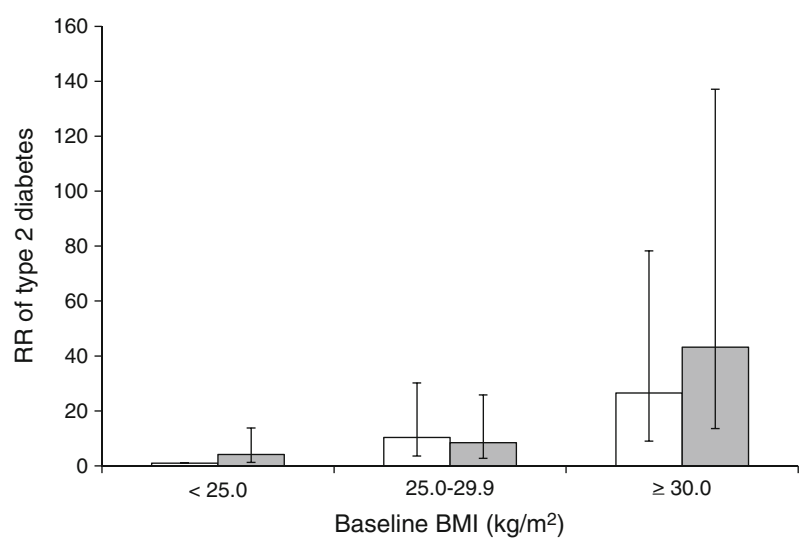

Fig. 2 Joint effect of baseline BMI and weight gain after GDM in association with long-term risk of progression from GDM to type 2 diabetes. White bars, weight gain $<5 \mathrm{~kg}$ after GDM; grey bars, weight gain $\geq 5 \mathrm{~kg}$ after GDM. The error bars indicate $95 \%$ CIs. The reference group was women who had a BMI $<25.0 \mathrm{~kg} / \mathrm{m}^{2}$ at baseline and gained $<5 \mathrm{~kg}$ after GDM (including weight loss and no change). Covariates in the multivariable model include: age (months), age at first birth (12-24, 25-29 or $\geq 30$ years), race/ethnicity (white, African-American, Hispanic, Asian, others), family history of diabetes (yes, no), oral contraceptive use (current, former, never), menopausal status (premenopausal, postmenopausal), change in cigarette smoking status (never to never, never to current, past to past, past to current, current to past, current to current), parity at baseline $(1,2,3+)$, change in parity $(0,1,2+)$, and baseline and changes (all in quartiles unless otherwise specified) in breastfeeding duration $(<1,1-5,6-11, \geq 12$ months), total energy intake, diet quality (i.e. AHEI score) and physical activity
GDM. Moreover, we observed that weight gain after GDM was significantly associated with risk of type 2 diabetes. These associations were independent of other major risk factors of type 2 diabetes, including lifestyle factors such as diet and physical activity.

The associations of BMI and weight change with risk of type 2 diabetes among women with a history of GDM, a population at high risk of type 2 diabetes, have not been comprehensively examined. A subgroup analysis of the Diabetes Prevention Program (DPP) randomised clinical trial showed that intensive lifestyle intervention including diet, physical activity and weight loss reduced the incidence of type 2 diabetes over 3 years among women with impaired glucose tolerance and a history of GDM [32]. Although another analysis from the overall DPP population showed that weight loss was the predominant predictor of diabetic risk reduction, independently of diet and physical activity [33], it was unclear whether that was the case in a high-risk population who may have reduced insulin secretion and/or chronic insulin resistance before pregnancy, i.e. women with a history of GDM. In the present study, we observed significant associations of BMI and weight change with the risk of progression from GDM to type 2 diabetes, which were independent of a healthful dietary pattern and physical activity, two major modifiable risk factors that have been inversely associated with risk of type 2 diabetes among women with a history of GDM [34, 35]. Furthermore, our findings support the recent call to action from the NDEP which underlines the importance of postpartum weight management after GDM [17].

Our findings were consistent with those of previous reports among women with a history of GDM that examined the associations of risk of type 2 diabetes with baseline BMI before pregnancy or shortly after the GDM pregnancy [36-41], 
most recent BMI [42] and weight change since baseline [37, $40,43]$. With longitudinal data on body weight, type 2 diabetic status and other major diabetic risk factors, which may be potential confounders, during 18 years of follow-up, our study had the unique opportunity to comprehensively examine the long-term risk of exposures including BMI, weight change and their joint effects on progression from GDM to type 2 diabetes. Our findings highlight the importance of controlling both initial weight and weight gain in the prevention of type 2 diabetes among women with a history of GDM.

Strengths of this study include its prospective cohort design, long-term follow-up, the high response rate of each questionnaire cycle, and comprehensive data that were longitudinally collected over decades. In addition, the NHS II participants are registered nurses, reducing potential confounding by educational attainment or differential access to healthcare. We acknowledge that there are limitations. First, body weight was self-reported in this study. However, self-reported weight was previously validated against technician-measured weight with a high correlation $(r=0.97)$ among a subset of NHS cohort participants [22]. Nonetheless, because of the prospective nature of the study design, misclassifications of the exposure would be non-differential. As such, the observed associations of BMI and weight gain with type 2 diabetes were more likely to be underestimated. The outcome, physician-diagnosed type 2 diabetes, was also on the basis of self-report. However, it was confirmed by supplemental questionnaires including diabetes-related symptoms, glucose testing and glucoselowering treatments. Specifically, in the study population of US female nurses, $98 \%$ of self-reported type 2 diabetic events were confirmed by medical records [31]. Second, BMI may not be able to accurately define obesity, in particular body fat distribution [44]. However, a previous meta-analysis of 32 studies has shown that although the clinical perspective focusing on central obesity is appealing, BMI and central obesity indicators have similar associations with incident diabetes [45]. Third, our study population consists mostly of white American women, thus the generalisation of our findings to other ethnic groups needs further evaluation. Fourth, screening bias may exist, because women who were more healthconscious, and therefore visited a physician more regularly, might have been more likely to receive a medical diagnosis than those who were less health-conscious. However, we found similar results in our sensitivity analyses restricting cases to symptomatic type 2 diabetes, minimising concerns for this bias.

In conclusion, among women with a history of GDM, we observed significant and positive associations of risk of type 2 diabetes with initial BMI within 2 years after diagnosis of GDM, the most recent BMI before diagnosis of type 2 diabetes and weight gain after GDM. Our findings provide evidence to support the recent call from NDEP and highlight the importance of achieving and maintaining a healthy weight in these high-risk women to prevent future development of type 2 diabetes.

Funding This study was supported by the Intramural Research Program of the Eunice Kennedy Shriver National Institute of Child Health and Human Development, National Institutes of Health (contract no. HHSN275201000020C). NHS II was funded by research grants DK58845, CA50385, P30 DK46200 and UM1 CA176726 from the National Institutes of Health. DKT was supported by a mentored fellowship from the ADA (no. 7-12-MN-34).

Duality of interest The authors declare that there is no duality of interest associated with this manuscript.

Contribution statement WB contributed to the design and analysis of the study and wrote the manuscript. DKT contributed to the data analysis and reviewed and edited the manuscript. EY, FBH, AAV, JEC, JLM, LGG, KB, SHL, MK and SFO interpreted the results and reviewed and edited the manuscript. $\mathrm{CZ}$ contributed to the design of the study and reviewed and edited the manuscript. $\mathrm{WB}$ and $\mathrm{CZ}$ are the guarantors of this work and, as such, had full access to all the data in the study and take responsibility for the integrity of the data and the accuracy of the data analysis. All authors approved the final version of the paper.

\section{References}

1. American Diabetes Association (2004) Gestational diabetes mellitus. Diabetes Care 27(Suppl 1):S88-S90

2. Buchanan TA, Xiang AH, Page KA (2012) Gestational diabetes mellitus: risks and management during and after pregnancy. Nat Rev Endocrinol 8:639-649

3. Kim C (2014) Maternal outcomes and follow-up after gestational diabetes mellitus. Diabet Med 31:292-301

4. Bellamy L, Casas JP, Hingorani AD, Williams D (2009) Type 2 diabetes mellitus after gestational diabetes: a systematic review and meta-analysis. Lancet 373:1773-1779

5. Cheung NW, Byth K (2003) Population health significance of gestational diabetes. Diabetes Care 26:2005-2009

6. Ferrara A, Kahn HS, Quesenberry CP, Riley C, Hedderson MM (2004) An increase in the incidence of gestational diabetes mellitus: Northern California, 1991-2000. Obstet Gynecol 103:526-533

7. Getahun D, Nath C, Ananth CV, Chavez MR, Smulian JC (2008) Gestational diabetes in the United States: temporal trends 1989 through 2004. Am J Obstet Gynecol 198(525):e521-e525

8. Zhang F, Dong L, Zhang CP et al (2011) Increasing prevalence of gestational diabetes mellitus in Chinese women from 1999 to 2008. Diabet Med 28:652-657

9. Chen L, Magliano DJ, Zimmet PZ (2012) The worldwide epidemiology of type 2 diabetes mellitus - present and future perspectives. Nat Rev Endocrinol 8:228-236

10. Bentley-Lewis R (2009) Gestational diabetes mellitus: an opportunity of a lifetime. Lancet 373:1738-1740

11. Colditz GA, Willett WC, Rotnitzky A, Manson JE (1995) Weight gain as a risk factor for clinical diabetes mellitus in women. Ann Intern Med 122:481-486

12. Ford ES, Williamson DF, Liu S (1997) Weight change and diabetes incidence: findings from a national cohort of US adults. Am J Epidemiol 146:214-222

13. Resnick HE, Valsania P, Halter JB, Lin X (2000) Relation of weight gain and weight loss on subsequent diabetes risk in overweight adults. J Epidemiol Community Health 54:596-602 
14. Will JC, Williamson DF, Ford ES, Calle EE, Thun MJ (2002) Intentional weight loss and 13-year diabetes incidence in overweight adults. Am J Public Health 92:1245-1248

15. Langenberg C, Sharp SJ, Schulze MB et al (2012) Long-term risk of incident type 2 diabetes and measures of overall and regional obesity: the EPIC-InterAct case-cohort study. PLoS Med 9:e1001230

16. Ratner RE (2007) Prevention of type 2 diabetes in women with previous gestational diabetes. Diabetes Care 30(Suppl 2):S242-S245

17. Gabbe SG, Landon MB, Warren-Boulton E, Fradkin J (2012) Promoting health after gestational diabetes: a National Diabetes Education Program call to action. Obstet Gynecol 119:171-176

18. Ferrara A, Hedderson MM, Albright CL et al (2011) A pregnancy and postpartum lifestyle intervention in women with gestational diabetes mellitus reduces diabetes risk factors: a feasibility randomized control trial. Diabetes Care 34:1519-1525

19. Baptiste-Roberts K, Barone BB, Gary TL et al (2009) Risk factors for type 2 diabetes among women with gestational diabetes: a systematic review. Am J Med 122(207-214):e204

20. Zhang C, Hu FB, Olsen SF et al (2014) Rationale, design, and method of the Diabetes \& Women's Health study - a study of long-term health implications of glucose intolerance in pregnancy and their determinants. Acta Obstet Gynecol Scand 93:1123-1130

21. Solomon CG, Willett WC, Carey VJ et al (1997) A prospective study of pregravid determinants of gestational diabetes mellitus. JAMA 278:1078-1083

22. Rimm EB, Stampfer MJ, Colditz GA, Chute CG, Litin LB, Willett WC (1990) Validity of self-reported waist and hip circumferences in men and women. Epidemiology 1:466-473

23. National Institutes of Health (1998) Clinical guidelines on the identification, evaluation, and treatment of overweight and obesity in adults - the evidence report. Obes Res 6(Suppl 2):51S-209S

24. Willett WC, Reynolds RD, Cottrell-Hoehner S, Sampson L, Browne ML (1987) Validation of a semi-quantitative food frequency questionnaire: comparison with a 1-year diet record. J Am Diet Assoc $87: 43-47$

25. Willett WC, Sampson L, Stampfer MJ et al (1985) Reproducibility and validity of a semiquantitative food frequency questionnaire. Am J Epidemiol 122:51-65

26. Salvini S, Hunter DJ, Sampson L et al (1989) Food-based validation of a dietary questionnaire: the effects of week-to-week variation in food consumption. Int J Epidemiol 18:858-867

27. Chiuve SE, Fung TT, Rimm EB et al (2012) Alternative dietary indices both strongly predict risk of chronic disease. J Nutr 142:1009-1018

28. Wolf AM, Hunter DJ, Colditz GA et al (1994) Reproducibility and validity of a self-administered physical activity questionnaire. Int J Epidemiol 23:991-999

29. Expert Committee on the Diagnosis and Classification of Diabetes Mellitus (1997) Report of the Expert Committee on the Diagnosis and Classification of Diabetes Mellitus. Diabetes Care 20:1183-1197

30. National Diabetes Data Group (1979) Classification and diagnosis of diabetes mellitus and other categories of glucose intolerance. Diabetes 28:1039-1057
31. Manson JE, Rimm EB, Stampfer MJ et al (1991) Physical activity and incidence of non-insulin-dependent diabetes mellitus in women. Lancet 338:774-778

32. Ratner RE, Christophi CA, Metzger BE et al (2008) Prevention of diabetes in women with a history of gestational diabetes: effects of metformin and lifestyle interventions. J Clin Endocrinol Metab 93:4774-4779

33. Hamman RF, Wing RR, Edelstein SL et al (2006) Effect of weight loss with lifestyle intervention on risk of diabetes. Diabetes Care 29:2102-2107

34. Tobias DK, Hu FB, Chavarro J, Rosner B, Mozaffarian D, Zhang C (2012) Healthful dietary patterns and type 2 diabetes mellitus risk among women with a history of gestational diabetes mellitus. Arch Intern Med 172:1566-1572

35. Bao W, Tobias DK, Bowers K et al (2014) Physical activity and sedentary behaviors associated with risk of progression from gestational diabetes mellitus to type 2 diabetes mellitus: a prospective cohort study. JAMA Intern Med 174:1047-1055

36. Lee AJ, Hiscock RJ, Wein P, Walker SP, Permezel M (2007) Gestational diabetes mellitus: clinical predictors and long-term risk of developing type 2 diabetes: a retrospective cohort study using survival analysis. Diabetes Care 30:878-883

37. Liu H, Zhang C, Zhang S et al (2014) Prepregnancy body mass index and weight change on postpartum diabetes risk among gestational diabetes women. Obesity (Silver Spring) 22:1560-1567

38. Schaefer-Graf UM, Klavehn S, Hartmann R et al (2009) How do we reduce the number of cases of missed postpartum diabetes in women with recent gestational diabetes mellitus? Diabetes Care 32:1960-1964

39. Pirkola J, Pouta A, Bloigu A et al (2010) Prepregnancy overweight and gestational diabetes as determinants of subsequent diabetes and hypertension after 20-year follow-up. J Clin Endocrinol Metab 95:772-778

40. Egeland GM, Meltzer SJ (2010) Following in mother's footsteps? Mother-daughter risks for insulin resistance and cardiovascular disease 15 years after gestational diabetes. Diabet Med 27:257-265

41. Ekelund M, Shaat N, Almgren P, Groop L, Berntorp K (2010) Prediction of postpartum diabetes in women with gestational diabetes mellitus. Diabetologia 53:452-457

42. Cho NH, Jang HC, Park HK, Cho YW (2006) Waist circumference is the key risk factor for diabetes in Korean women with history of gestational diabetes. Diabetes Res Clin Pract 71:177-183

43. Peters RK, Kjos SL, Xiang A, Buchanan TA (1996) Long-term diabetogenic effect of single pregnancy in women with previous gestational diabetes mellitus. Lancet 347:227-230

44. Okorodudu DO, Jumean MF, Montori VM et al (2010) Diagnostic performance of body mass index to identify obesity as defined by body adiposity: a systematic review and meta-analysis. Int $\mathrm{J}$ Obes 34:791-799

45. Vazquez G, Duval S, Jacobs DR Jr, Silventoinen K (2007) Comparison of body mass index, waist circumference, and waist/ hip ratio in predicting incident diabetes: a meta-analysis. Epidemiol Rev 29:115-128 